\title{
Formation of Cyclophane Macrocycles in Carbazole-Based Biradicaloids: Impact of the Dicyanomethylene Substitution Position
}

Irene Badía-Domínguez, ${ }^{\dagger}$ Andrés Pérez-Guardiola, ${ }^{\ddagger}$ Juan Carlos Sancho-García, ${ }^{\ddagger} \bullet$

Juan T. López Navarrete, ${ }^{\dagger}$ Víctor Hernández Jolín, ${ }^{\dagger}$ Hongxiang Li, ${ }^{\S \odot}$ Daisuke Sakamaki, ${ }^{\| \oplus ~ S h u ~ S e k i,},{ }$ and M. Carmen Ruiz Delgado $* \dagger \odot$

${ }^{\dagger}$ Department of Physical Chemistry, University of Malaga, Campus de Teatinos s/n, 29071 Malaga, Spain

${ }^{\ddagger}$ Department of Physical Chemistry, University of Alicante, 03080 Alicante, Spain

${ }^{\S}$ Key Laboratory of Synthetic and Self-Assembly Chemistry for Organic Functional Materials, Shanghai Institute of Organic Chemistry, Chinese Academy of Sciences, No. 345 Lingling Road, Shanghai 200032, China

"Department of Molecular Engineering, Graduate School of Engineering, Kyoto University, Nishikyo-ku, Kyoto 615-8510, Japan

\section{Supporting Information}

ABSTRACT: We have recently demonstrated that carbazolebased biradicaloids are promising building blocks in dynamic covalent chemistry. To elucidate their intriguing dynamic covalent chemical properties, it is necessary to understand the physical origin of their biradical nature. To this end, here we focus on two quinoid carbazole systems substituted with dicyanomethylene (DCM) groups via para ( $\boldsymbol{p}$-Cz-alkyl) or meta positions $(\boldsymbol{m}-\mathbf{C z}-\mathbf{p h})$, which are able to form cyclophane macrocycles by the formation of long $\mathrm{C}-\mathrm{C}$ bonds between the bridgehead carbon atoms linked to the DCM groups. We aim at exploring the following questions: (i) How is the biradicaloid character of a quinoid carbazole affected by the substitution position of the DCM groups? (ii) How is the stability of the resulted cyclophane aggregate attained? (iii) How is the dynamic interconversion between the carbazole-based monomers and cyclophane aggregates affected by this subtle change in the substitution pattern position? Density functional theory-based calculations reveal that both $\mathbf{p}$-Cz-alkyl and $\mathbf{m}-\mathbf{C z}$-ph are open-shell biradicals in the ground electronic state, with the DCM substitution in the meta position resulting in a more pronounced biradical character. In contrast, the derivatization via the nitrogen of the carbazole unit is not predicted to affect the biradicaloid character. The spontaneous nature of the cyclophane-based macrocycle formation (i.e., the cyclic tetramer in $\mathbf{p}$-Cz-alkyl and the cyclic trimer and the tetramer in $\boldsymbol{m}$-Czph) is supported by the negative relative Gibbs free energies calculated at $298 \mathrm{~K}$. Interestingly, cyclic oligomers in which the DCM groups are inserted in the meta position tend to adopt folded conformations with attractive $\pi-\pi$ interactions resulting in more stable aggregates; in contrast, note that an extended ring-shaped conformation is acquired for $(\boldsymbol{p} \text {-Cz-alkyl) })_{4}$. In addition, the larger spin density on the bridgehead carbon atom in the meta-substituted system strengthens the bridging $\mathrm{C}-\mathrm{C}$ bond in the aggregate forms, hampering its dissociation. In fact, the $\mathrm{C}-\mathrm{C}$ bond dissociation of $(\boldsymbol{m}-\mathbf{C z}-\mathbf{p h})_{4}$ and $(\boldsymbol{m}-\mathbf{C z} \text {-ph })_{3}$ was suppressed in solution state, although it was achieved in solid state in response to soft external stimuli (i.e., temperature and grinding). In summary, we report a very comprehensive study aiming at elucidating the challenging chemical properties of carbazole-based biradicaloid systems.

\section{INTRODUCTION}

Recently, the development of the chemistry of $\pi$-conjugated biradical species has attracted much interest because of their unique optical, ${ }^{1}$ electronic, ${ }^{2}$ and magnetic properties. ${ }^{3}$ According to IUPAC, a biradical is described as a molecular entity with two radical centers (possibly delocalized), which act nearly independently of each other. ${ }^{4}$ In other words, systems characterized by two unpaired electrons with a longdistance between them would result in distinct singlet and triplet electronic states because of the negligible interelectronic interaction. The elucidation of the electronic configuration of these systems is fundamental to understand their electronic and structural properties. ${ }^{5}$ However, it is possible that two radical centers interact significantly, and in this case, these species are better defined as biradicaloids according to the IUPAC Gold Book. ${ }^{4}$ These species have two representative resonance forms in the ground states: (i) a closed-shell (CS)

Received: December 5, 2018

Accepted: February 19, 2019

Published: March 4, 2019 
(a) $p$-Cz-alkyl

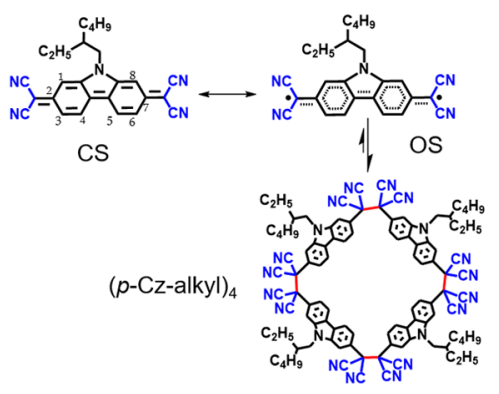

(b) $m$-Cz-ph

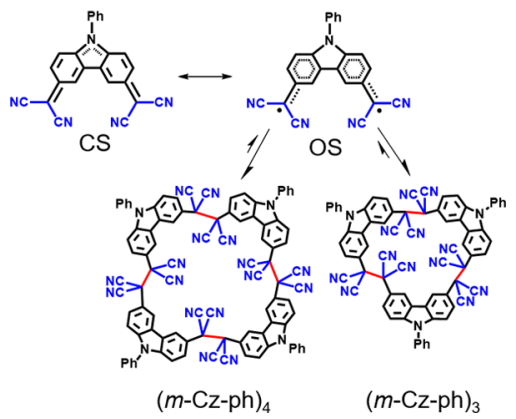

Figure 1. Equilibrium between the isolated monomers (a) $p$-Cz-alkyl and (b) $m$-Cz-ph and their corresponding cyclophane-type oligomers. The resonant structures of the ground state of the monomer corresponding to the closed-shell (CS) singlet state (left) and the open-shell (OS) singlet biradical state (right) are also shown.

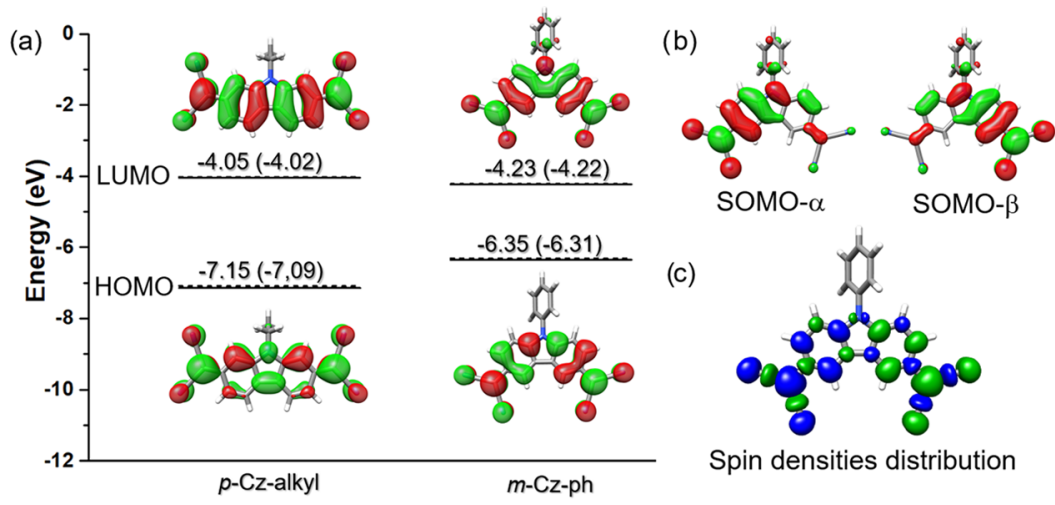

Figure 2. (a) DFT-calculated molecular orbital diagram for $\boldsymbol{p}$-Cz-alkyl and $\boldsymbol{m}$-Cz-ph. Values in parenthesis correspond to $\mathrm{N}$-substituted model analogues $p$-Cz-ph and $m$-Cz-alkyl. (b) Calculated singly occupied molecular orbital (SOMO) of $\alpha$ and $\beta$ electrons and (c) spin density distribution in the biradical singlet ground state of $m$-Cz-ph. The blue and green surfaces represent $\alpha$ and $\beta$ spin densities, respectively. All of the values are obtained at the M06-2X/6-31G** level.

quinoid structure and (ii) an open-shell (OS) singlet biradical structure. ${ }^{6}$

Interestingly, it has been recently demonstrated that several biradical systems are able to form long $\mathrm{C}-\mathrm{C}$ bonds between unpaired electrons from the radical centers of the monomers resulting in macrocycles, ${ }^{7}$ staircase oligomers, ${ }^{8}$ or polymers by diradical polymerization. ${ }^{9}$ Thus, $\pi$-conjugated biradical compounds have emerged as essential building blocks in dynamic covalent chemistry (DCC). ${ }^{10}$ This field is focused on the creation of structural scaffolds based on chemical components that interact through strong but reversible bonds. In fact, dynamic covalent bonds receive a lot of attention because of their unique feature to become reversible under mild conditions. $^{11}$

Carbazole-based materials have been deeply studied due to their good charge-transport properties and chemical stabilities. ${ }^{12}$ Recently, we investigated one of the smallest quinoidal biradicaloids reported to be stable in solution under ambient conditions, a carbazole-based system substituted with dicyanomethylene groups via 2,7 (para) positions ( $\boldsymbol{p}$-Cz-alkyl in Figure 1). This quinoidal system is able to form a macrocycle cyclophane-type tetramer $(\boldsymbol{p} \text {-Cz-alkyl })_{4}$ by the formation of long $\sigma$-bonds between the central carbon atoms linked to the dicyanomethylene groups. A dynamic interconversion is demonstrated between the isolated monomer and cyclic tetramer in response to soft external stimuli (temperature, pressure, and light), all causing strong chromic features. ${ }^{13}$ The reversibility of this mechanism has been demonstrated by combined IR, Raman, electron paramagnetic resonance, and ${ }^{1} \mathrm{H}$ NMR spectral data, with support from density functional theory (DFT) calculations. The single-crystal X-ray analysis demonstrated that the macrocycle cyclophane is formed by four molecules joined together by coupling of eight unpaired electrons creating a structural cavity of $12.52 \AA^{13}$

A similar carbazole compound with terminal dicyanomethylene (DCM) groups but meta-substituted has also been recently demonstrated to form macrocyclic cyclophanetype oligomers (i.e., a trimer and a tetramer) by self-assembly of the monomers having two radicals. ${ }^{14}$ Therefore, these two quinoid carbazole-based systems substituted with DCM groups via 2,7 (para) or 3,6 (meta) positions ( $\boldsymbol{p}$-Cz-alkyl and $\boldsymbol{m}$-Czph, respectively; see Figure 1) represent a very good test case to study the impact of the substitution pattern on the selfassembly formation of stimuli-responsive cyclophanes.

Specifically, we will focus here on how the different substitution positions of the DCM groups in carbazole-based quinoid systems affect the following points: (i) the ground electronic state properties of the monomer (i.e., biradicaloid character), (ii) the spatial (or supramolecular) arrangement of the formed cyclophane aggregates, and (iii) the dynamic character of the monomer/cyclophane transformation both in solution and solid state in response to external stimuli (temperature and pressure). To this end, we use electronic absorption spectroscopy (UV-vis-NIR) and vibrational spectroscopy (Raman and IR) in combination with state-ofthe-art DFT calculations. 


\section{RESULTS AND DISCUSSION}

2.1. Ground-State Electronic Structures and Biradical Character of the Monomers. We first investigate the ground-state electronic structure and biradical character of the carbazole-based molecules. Among these, we analyze their optimized geometries, singlet-triplet energy gap $\left(\Delta E_{S-T}\right)$, and highest occupied molecular orbital (HOMO)-lowest unoccupied molecular orbital (LUMO) energetics. Two additional theoretical models are included in the discussion ( $p$-Cz-ph and $m$-Cz-alkyl, see Figure S1) to investigate the role played by the $\mathrm{N}$-substitution (i.e., alkyl vs phenyl groups). The calculated energy diagram of the frontier molecular orbitals shows that the quinoid carbazole-based systems exhibit a very small HOMO-LUMO gap due to the strong stabilization of the LUMO upon the insertion of electron-withdrawing DCM groups (see Figures 2a and S2). An additional HOMOLUMO gap decrease of $\sim 1 \mathrm{eV}$ is also found when comparing the para-substituted systems with the meta-substituted homologues (i.e., $3.10 \mathrm{eV}$ in $p$-Cz-alkyl and $2.09 \mathrm{eV}$ in $m$ Cz-alkyl), whereas $\mathrm{N}$-substitution has a negligible effect on the energy levels (i.e., $3.07 \mathrm{eV}$ in $\boldsymbol{p}$-Cz-ph and $2.12 \mathrm{eV}$ in $\boldsymbol{m}$-Czph). The HOMO and LUMO from the closed-shell configuration are largely delocalized over the $\pi$-conjugated core showing a large spatial overlap on the DCM groups, thus representing the more reactive sites that would lead to a pronounced biradical character. In fact, DFT-calculated data reveals that DCM-substituted carbazoles are open-shell singlet biradicals in the ground state, with this effect being more significant when the DCM groups are connected at the meta position (see Table S1). The energy difference between the open-shell (OS) and closed-shell (CS) states is $\sim 2 \mathrm{kcal} / \mathrm{mol}$ for the para-substituted systems, whereas this difference increases up to $\sim 13 \mathrm{kcal} / \mathrm{mol}$ in the meta-substituted homologues. In addition, the singlet-triplet energy gap decreases roughly from 5 to $1.2 \mathrm{kcal} / \mathrm{mol}$ on going from the para- to the meta-substituted carbazoles. The energetics trend of the ground electronic states is not affected by the amount of the exact exchange included in the M06 suite of functionals used (see Table S2).

Interestingly, the calculated data also reveals the following results: (i) The singly occupied molecular orbital (SOMO) profiles of the $\alpha$ and $\beta$ spins show a typical disjoint feature (see Figure $2 \mathrm{~b}$ for $\boldsymbol{m}$-Cz-ph and Figure S3 for the rest of compounds). (ii) The spin densities are distributed along the whole $\pi$-conjugated backbone with the carbon atoms linked to the $\mathrm{CN}$ groups having the largest density (see Figure $2 \mathrm{c}$ for $\boldsymbol{m}$ Cz-ph and Figure S4 for the rest of compounds). This effect is more significant when the DCM groups are attached at the meta positions (i.e., values of -0.47 and -0.55 for the carbon bridgehead atoms of $\boldsymbol{p}$-Cz-ph and $\boldsymbol{m}$-Cz-alkyl, respectively). Moreover, the spin density over the carbazole core is moderately larger in meta- than in para-substituted systems indicating that $\pi$-conjugation of the unpaired electrons on the bridgehead carbons of the DCM groups with the central carbazole unit is facilitated and thus the open-shell biradical species is favored (see Figure 3). (iii) As in the case of the para-substituted systems, ${ }^{13}$ aromatization of the phenyl groups of the carbazole units is also the driving force for the stabilization of the biradical species in the meta-substituted systems (i.e., the closed shell shows a quinoidal pattern, whereas the open-shell form displays a certain degree of aromatization; see DFT-calculated bond lengths in Figure 3).

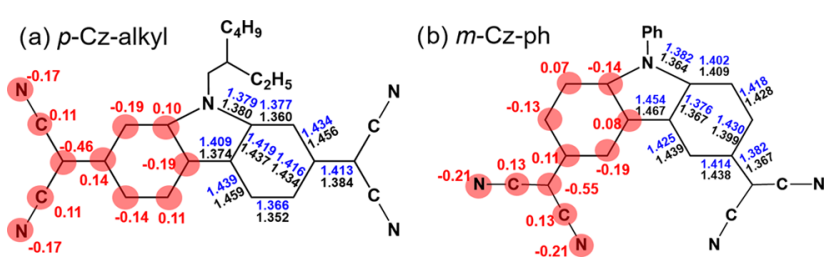

Figure 3. DFT-calculated (M06-2X/6-31G** level) bond lengths ( $)$ for the closed-shell (black) and open-shell singlet (blue) biradical forms of the ground electronic state of (a) $p$-Cz-alkyl and (b) $m$-Czph. The numbers given in red denote the spin population of the specific carbon and nitrogen atoms.

However, there is a weakening of the conjugated double bonds over the whole carbazole backbone (both phenyl and pyrrole rings) upon the insertion of DCM groups at the meta position, therefore facilitating its rupture to create an open-shell biradical.

The calculated spin density on the bridgehead carbon atoms linked to the $\mathrm{CN}$ groups suggests a stronger intermolecular $\mathrm{C}-$ $\mathrm{C}$ binding in the $\boldsymbol{m}$-Cz-ph monomers than in the $\boldsymbol{p}$-Cz-alkyl analogues, in agreement with the data discussed below. Similar intermolecular bonding characteristics are discussed based on the conventional Hammett para-substituent parameters in a series of aryl dicyanomethyl radicals. ${ }^{15}$

For a better understanding of the key structure-biradical character relationship, the biradical character has been calculated using two methods (see Figure 4 for $p$-Cz-alkyl (a)

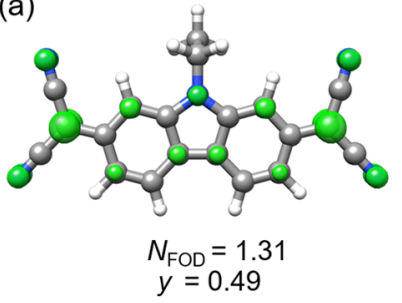

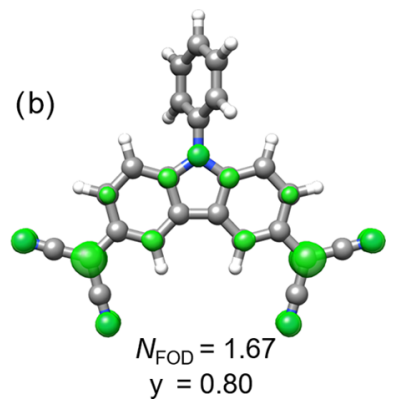

Figure 4. Plots of the FOD density $\left(\sigma=0.005 e \mathrm{bohr}^{-3}\right)$ and calculated $N_{\text {FOD }}$ values for (a) $p$-Cz-alkyl and (b) $m$-Cz-ph, as obtained from the FT-DFT method. The biradical character (given by $y$ values) is also shown.

and $m$-Cz-ph and Figure $S 5$ for the rest of compounds). First, the biradical character $y(0<y<1$, where $y=0$ represents a closed-shell state and $y=1$ represents a pure biradical state) is calculated from the occupation numbers of the highest occupied natural orbital and the lowest unoccupied natural orbital using the spin-unrestricted Hartree-Fock (HF) scheme. ${ }^{16} \mathrm{~A}$ pronounced increase of the biradical character is predicted for the meta-substituted compounds (i.e., $y=0.80$ for $m$-Cz-ph) when compared to the para-substituted homologues (i.e., $y=0.51$ for $p$-Cz-ph). However, Nsubstitution has a negligible effect on the biradical character (i.e., $y=0.80$ for $\boldsymbol{m}$-Cz-ph and 0.79 for $\boldsymbol{m}$-Cz-alkyl). Second, the fractional orbital density (FOD) method that incorporates a strong correlation effect has also been used to calculate the $N_{\text {FOD }}$ values. The $N_{\text {FOD }}$ values are the integrated number of electrons arising from the fractional occupation of orbitals and represent a very good estimation of the biradical character. Following the trend reported before for a set of some 
polycyclic aromatic molecules, ${ }^{17}$ where a linear relationship between the $N_{\mathrm{FOD}}$ values and the experimental biradical character is reported (see Figure S6), $N_{\text {FOD }} \geq 1.5$ indicates a highly pronounced biradical character, whereas $N_{\mathrm{FOD}}<1.5$ is associated with a slightly pronounced biradical character. The FOD plots for the carbazole-based systems show that the spatial distribution of the unpaired electrons is highly delocalized over the whole molecule with a strong contribution from the central carbon atoms of the DCM groups (see Figure 4). The $N_{\text {FOD }}$ values calculated for the meta-substituted systems $\left(N_{\mathrm{FOD}} \sim 1.7\right)$ indicate a moderate to strong biradical character, whereas the para-substituted homologues with $N_{\text {FOD }}$ values of 1.3 are situated on the borderline (i.e., similar values are reported for linear acenes such as hexacene); see Figure S6. Thus, our calculations suggest that the biradical character is strongly affected by the DCM substitution position, whereas the N-substitution has a negligible effect.

2.2. DFT Insights into the Structures and Stability of the Cyclophane Macrocycles. Thanks to their unique biradicaloid character, the quinoid carbazole systems are highly prone to form cyclic oligomers via $\sigma$-oligomerization reactions through the formation of long CC bonds between the carbon atoms linked to the $\mathrm{CN}$ groups. For instance, it has already been demonstrated that $\boldsymbol{p}$-Cz-alkyl monomers form a cyclic tetramer ( $\boldsymbol{p}$-Cz-alkyl $)_{4}$ and the $\boldsymbol{m}$-Cz-ph monomers can build a cyclic trimer $(\boldsymbol{m}-\mathbf{C z}-\mathbf{p h})_{3}$ and a cyclic tetramer $(\boldsymbol{m}-\mathbf{C z}-$ $\mathbf{p h})_{4} \cdot{ }^{13,14}$ The structures of $(\boldsymbol{p} \text {-Cz-alkyl })_{4}$ and $(\boldsymbol{m} \text {-Cz-ph })_{3}$ were resolved by X-ray analysis, showing that the $\mathrm{C}-\mathrm{C}$ bonds between the monomers $\left[1.608-1.639 \AA\right.$ for $(m-\mathbf{C z}-\mathbf{p h})_{3}$ and $1.631 \AA$ for $\left.(p \text {-Cz-alkyl })_{4}\right]$ were remarkably longer than typical $\mathrm{C}\left(\mathrm{sp}^{3}\right)-\mathrm{C}\left(\mathrm{sp}^{3}\right)$ bonds $(1.54 \AA)$. Figure 5a,b displays a side-byside comparison between the DFT-calculated and experimental structures of $(\boldsymbol{p}-\mathbf{C z} \text {-alkyl })_{4}$ and $(\boldsymbol{m}-\mathbf{C z}-\mathbf{p h})_{3}$ (for the comparison of bond lengths, see Figures S7 and S8). The computed global minima are in good agreement with the

(a) $(p-C z-a l k y \mid)_{4}$
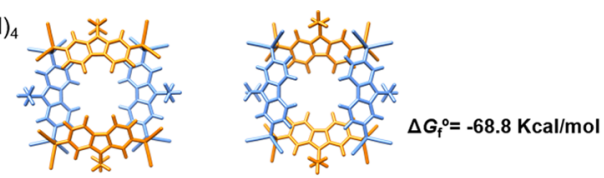

(b) $(m-\mathrm{Cz}-\mathrm{ph})_{3}$
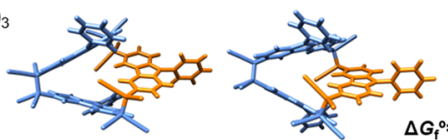

$\Delta G_{\mathrm{f}}^{\circ}=-59.4 \mathrm{Kcal} / \mathrm{mol}$

(c) $(m-\mathrm{Cz}-\mathrm{ph})_{4}$
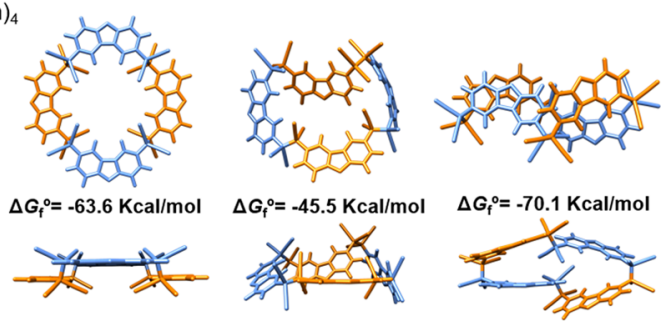

Figure 5. X-ray (left) and DFT-computed global minimum structure (right) for (a) ( $p$-Cz-alkyl $)_{4}$ and (b) $(\boldsymbol{m} \text {-Cz-ph })_{3}$ cyclic oligomers. (c) Top view (up) and side view (down) of DFT-computed global minimum structure for $(\boldsymbol{m} \text {-Cz-ph })_{4}$ cyclic oligomers in three different configurations: ring-shaped (left), distorted ring-shaped (center), and folded (right). The $\mathrm{N}$-substituted phenyl groups have been omitted for clarity. Free energy of formation values (at $298 \mathrm{~K}$ ) calculated at the M06-2X/6-31G** level are also shown. experimental X-ray data, showing that the cyclic tetramer ( $p$ Cz-alkyl $)_{4}$ is formed by four molecules extended in the cavity periphery (with negligible $\pi-\pi$ overlap) creating a ring-shaped structural cavity of $12.52 \AA$. In contrast, a folded molecular shape conformation is formed by the cyclic trimer $(\boldsymbol{m}-\mathbf{C z}-\mathbf{p h})_{3}$ resulting in labile $\pi-\pi$ interactions for the two $\mathrm{Cz}$ units that point in the same direction (see Figure S9a). The negative free energies of formation calculated at $298 \mathrm{~K}$ reveal the spontaneous nature of the oligomerization reactions. Thus, these cyclophane-type cyclic oligomers are expected to be formed at room temperature (RT), in good agreement with experiments.

Unfortunately, we could not obtain single crystals of $(\boldsymbol{m}-\mathbf{C z}-$ ph $)_{4}$. Here, we performed DFT calculations for different conformations to address the most favorable intermolecular arrangement of this aggregate. To this end, three different configurations for the spatial disposition of the $\mathrm{Cz}$ fragments were considered: (i) a ring-shaped configuration with the four $\mathrm{Cz}$ units extended over the periphery with the $\mathrm{N}$-phenyl groups pointed outward of the cavity, (ii) a distorted ring-shaped configuration where one of the $\mathrm{Cz}$ fragments is twisted with its $\mathrm{N}$-phenyl group pointed toward the inside of the ring cavity, and (iii) a folded structure where the $\mathrm{Cz}$ units are co-facially reoriented in a pairwise configuration. The calculated free energies reveal that the formation of the folded configuration is the most favorable (i.e., about 7 and $25 \mathrm{kcal} / \mathrm{mol}$ more stable than the ring-shaped and the distorted ring-shaped configurations, respectively). This can be explained by the presence of co-facially superimposed $\mathrm{Cz}$ units in the folded configuration resulting in favorable $\pi-\pi$ interactions, which would, in turn, increase the dispersion attractive term of the interaction energy. For instance, $\pi-\pi$ distances of $3.54 \AA$ are predicted between adjacent phenyl rings linked to the $\mathrm{C}-\mathrm{C} \sigma$-bonds in $(\boldsymbol{m} \text {-Cz-ph })_{4}$; see Figure S9b. In addition, the difference of the spin density on the carbon atoms linked to the $\mathrm{CN}$ groups, which is larger in $\boldsymbol{m}$-Cz-ph than in $\mathbf{p}$-Cz-alkyl, as discussed above, would also strengthen the $\mathrm{C}-\mathrm{C}$ bond bridging in $(\boldsymbol{m}$ Cz-ph) . $^{2}$

In summary, our results indicate that changing the DCM groups in the carbazole unit from the para to meta position results in a profound change of the macrocycle conformation. Thus, the resulting cyclic oligomers become more stable leading to stronger $\mathrm{C}-\mathrm{C}$ bonds when compared to the parasubstituted homologues. These observations anticipate that the insertion of the DCM groups at the meta position would disfavor the $\mathrm{C}-\mathrm{C}$ bond dissociation by some external stimuli when compared to the para-substituted homologues, as we will discuss below in more detail.

2.3. Interconversion between Cyclophane Aggregates and Carbazole-Based Monomers in Solution. We now explore the optical properties of the cyclophane aggregates and the possibility of $\mathrm{C}-\mathrm{C}$ bond dissociation in response to external stimuli (i.e., heat and time) to afford the isolated biradicaloid monomers. As seen in Figure $6 \mathrm{a}, \mathrm{b}$, the UV-vis absorption spectra at room temperature of both ( $p$ Cz-alkyl $)_{4}$ and $(\boldsymbol{m} \text {-Cz-ph })_{4}$ clearly show a group of bands below $\lambda=400 \mathrm{~nm}$, which correspond to the cyclophane tetramer. When the white powder of $(\boldsymbol{p} \text {-Cz-alkyl })_{4}$ was dissolved in chloroform, the colorless solution turned gradually purple with broad bands appearing between 650 and $850 \mathrm{~nm}$, which reveals the formation of the isolated biradicaloid monomers. ${ }^{13}$ In contrast, the solution of the white powder of $(m-\mathbf{C z}-\mathbf{p h})_{4}$ kept its initial transparent color and the 

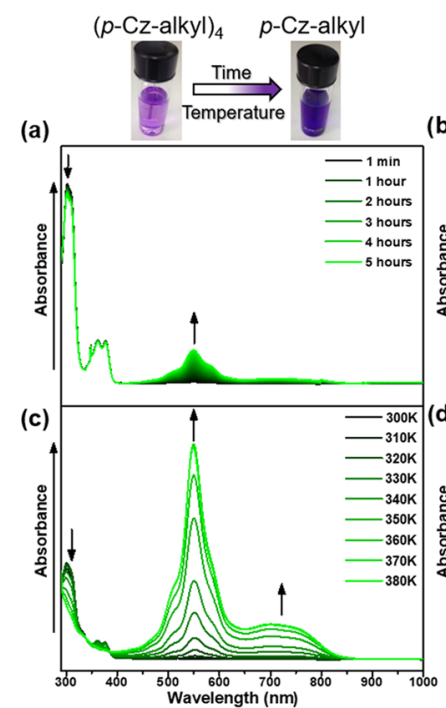

(b)

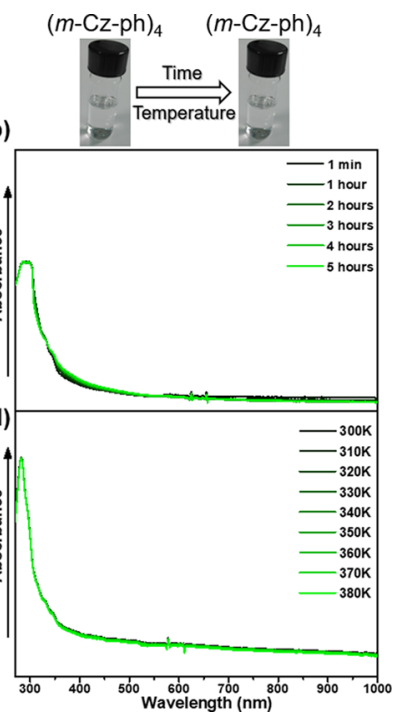

Figure 6. (a) UV-vis absorption changes in chloroform accompanying transformation of cyclophane tetramer $(p \text {-Cz-alkyl })_{4}$ to $p$-Czalkyl monomer at room temperature as a function of time. (b) UVvis absorption of a freshly prepared solution in chloroform of $(\mathbf{m}-\mathbf{C z}-$ $\mathbf{p h})_{4}$ at room temperature as a function of time. (c) UV-vis spectral changes accompanying conversion of cyclophane tetramer $(p-\mathbf{C z}-$ alkyl) $)_{4}$ to $p$-Cz-alkyl monomer upon heating from 300 to $380 \mathrm{~K}$. (d) UV-vis absorption of the solution of $(\boldsymbol{m} \text {-Cz-ph })_{4}$ in toluene as a function of temperature.

spectrum showed only the bands of the aggregate. We also investigate the tetramer-monomer dissociation in solution upon heating. Figure $6 c$,d shows the UV-vis-NIR absorption spectra in toluene at temperatures between 300 and $380 \mathrm{~K}$. For $(p \text {-Cz-alkyl })_{4}$, the intensities of the low-energy band strongly increased upon heating, thus showing the formation of the isolated biradical monomer. In contrast, no absorption spectral changes are found for $(\boldsymbol{m}-\mathbf{C z}-\mathbf{p h})_{4}$ after heating in toluene. Other solvents (o-dichlorobenzene and tetrahydrofuran) were checked upon increasing the temperature to favor the cyclophane dissociation, but, unfortunately, we could not obtain any further spectral changes even after heating up to 410 K (see Figure S10).

Therefore, in contrast to that of $(\boldsymbol{p} \text {-Cz-alkyl })_{4}, \mathrm{C}-\mathrm{C}$ bond dissociation of $(\boldsymbol{m}-\mathbf{C z}-\mathbf{p h})_{4}$ was suppressed in solution state (either upon heating or as a function of time). Similar behavior is also found for $(\boldsymbol{m}-\mathbf{C z}-\mathbf{p h})_{3}$ cyclic oligomer (see Figure S11). This data reveals that substitution of DCM groups at the meta position of carbazole increases the spin density on the bridgehead carbon atom and consequently strengthens the bridging $\mathrm{C}-\mathrm{C}$ bond in the aggregate form, hampering its dissociation. This is in good agreement with theoretical data that predict that meta-substitution results in more stable cyclic oligomers than substitution in the para position (see Figure 5). This trend observed in gas-phase calculations is even more pronounced when solvent effects are included using the polarizable continuum model (PCM) approach (see Table S3 in the Supporting Information).

2.4. Interconversion between Cyclophane Aggregates and Carbazole-Based Monomers in Solid State. We now use IR and Raman spectroscopies to monitor solidstate bond dissociation by mechanical stimuli (i.e., grinding with a mortar and pestle). Note that the colorless solid powders of $(\boldsymbol{m} \text {-Cz-ph })_{4}$ and $(\boldsymbol{m}-\mathbf{C z}-\mathbf{p h})_{3}$ cyclic oligomers turn to deep blue upon grinding, demonstrating the $\mathrm{C}-\mathrm{C}$ bond dissociation in solid state. ${ }^{14}$ A similar chromic effect from white to purple was previously found in $(\boldsymbol{p} \text {-Cz-alkyl })_{4}$ after grinding. ${ }^{1 \frac{1}{3}}$ Figure 7 compares the IR spectra of $(\boldsymbol{m}-\mathbf{C z}-\mathbf{p h})_{4}$ and $(p-C z-a l k y l)_{4}$ as a white powder and after grinding used to prepare the $\mathrm{KBr}$ pellet. For the white powder, an intense band appears at $2253 \mathrm{~cm}^{-1}$ related with the $\mathrm{CN}$ stretching, $\nu(\mathrm{CN})$, which is very similar to that observed in nonconjugated nitriles. However, three additional $\nu(\mathrm{CN})$ bands with low intensity appear at low frequencies $\left(2230,2214\right.$, and $\left.2190 \mathrm{~cm}^{-1}\right)$ in the meta-substituted $(\boldsymbol{m}-\mathbf{C z}-\mathbf{p h})_{4}$ cyclic oligomer; this might be related with the formation of staircase oligomers leaving two unpaired electrons at the extremity such as open linear tetramers $(\boldsymbol{m}-\mathbf{C z} \text {-ph })_{4}$-op, trimers $(\boldsymbol{m}-\mathbf{C z} \text {-ph })_{3}$-op, or dimers $(\boldsymbol{m} \text {-Cz-ph })_{2}$-op (note that during the recording of the attenuated total reflection (ATR) spectrum, the samples support some pressure). ${ }^{18}$ We performed DFT calculations

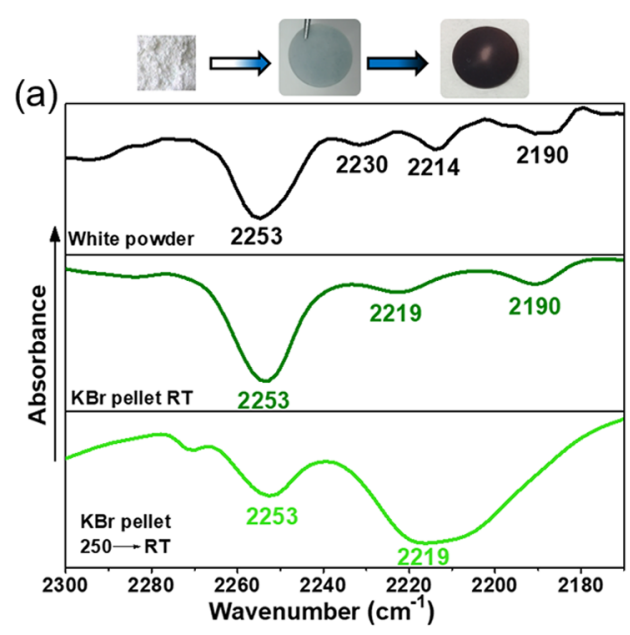

(b)

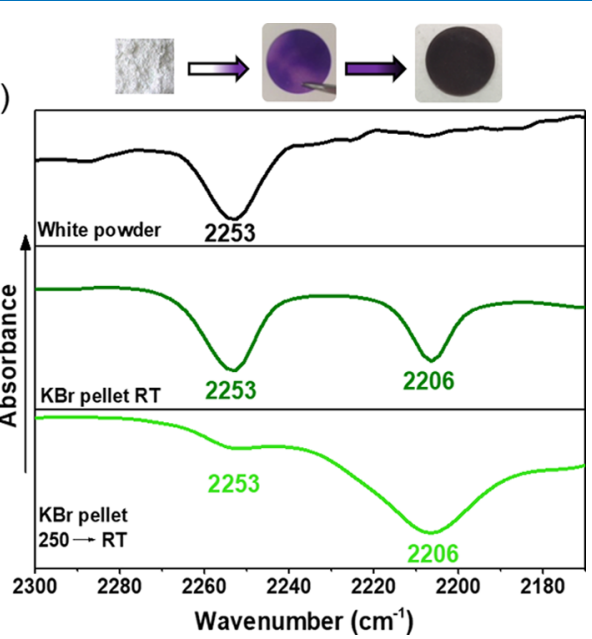

Figure 7. (a) IR spectrum of $(\boldsymbol{m} \text {-Cz-ph })_{4}$ as a white powder at room temperature (top); a deep blue $\mathrm{KBr}$ pellet containing a mixture of tetramer, open linear oligomers, and isolated biradical $m$-Cz-ph obtained after grinding at room temperature (middle); and the KBr pellet with dominant deep blue biradicals after heating and cooling to RT (bottom). (b) IR spectrum of ( $p$-Cz-alkyl) ${ }_{4}$ as a white powder at room temperature (top), a purple $\mathrm{KBr}$ pellet containing a mixture of tetramer and biradical $\boldsymbol{p}$-Cz-alkyl obtained after grinding at room temperature (middle), and the $\mathrm{KBr}$ pellet with dominant purple biradicals after heating and cooling to RT (bottom). 
(a)

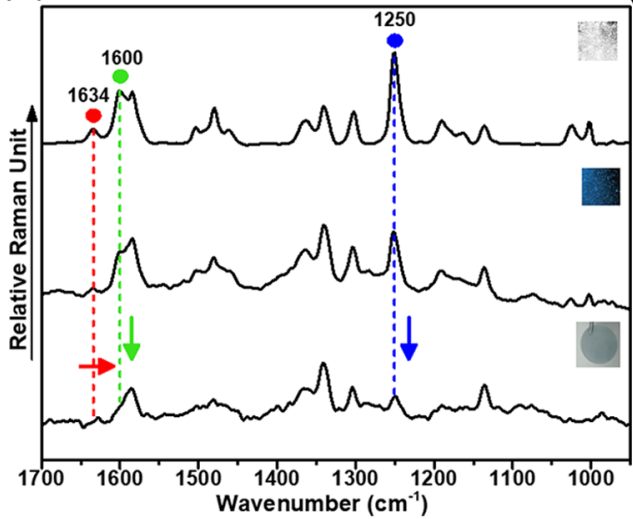

(b)

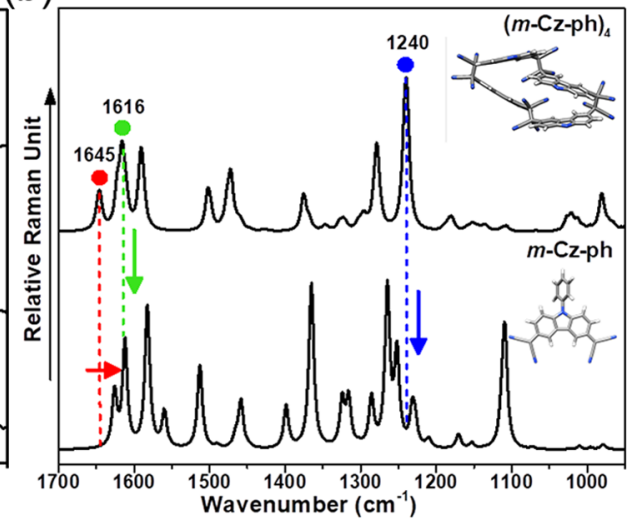

Figure 8. (a) FT-Raman spectra of $(\boldsymbol{m} \text {-Cz-ph })_{4}$ as a white powder at room temperature (top), a blue powder after grinding (middle), and a deep blue $\mathrm{KBr}$ pellet (bottom). (b) Theoretical Raman spectra (M06-2X/6-31G**) of cyclophane tetramer ( $m$-Cz-ph $)_{4}$ and $m$-Cz-ph monomer.

for the open linear oligomers revealing their spontaneous formation (see Figure S12); in addition, their calculated IR spectra are also in good accordance with the experimental spectral evolution (see Figure S13). Interestingly, the samples change color after grinding, and two $\nu(\mathrm{CN})$ bands with low intensities appear at 2219 and $2190 \mathrm{~cm}^{-1}$ for the metasubstituted system, whereas a very intense band at $2206 \mathrm{~cm}^{-1}$ rises for the para-substituted compound. This indicates the predominant formation of isolated biradicaloid monomers in the para-substituted system upon pressure application, ${ }^{13}$ whereas in the meta-substituted system, staircase oligomers of small size together with isolated monomers might also be formed [note that similar behavior is also found for $(\boldsymbol{m}-\mathbf{C z}$ ph) $)_{3}$ cyclic oligomer; see Figure S14]. Interestingly, after applying temperature of up to $250{ }^{\circ} \mathrm{C}$ and then cooling to room temperature, the isolated biradicaloid monomers are preferentially formed in both the meta- and para-substituted systems as suggested by the strong increase of the $\nu(\mathrm{CN})$ bands at 2219 and $2206 \mathrm{~cm}^{-1}$, respectively, related with the isolated monomers. However, the pronounced prevalence of the $\nu(\mathrm{CN})$ band at $2253 \mathrm{~cm}^{-1}$ in the meta-substituted system when compared to the para-substituted analogue indicates that $\sigma$-oligomers formed by $\boldsymbol{m}$-Cz-ph fragments are harder to dissociate.

Figure 8 shows the FT-Raman spectra of $(\boldsymbol{m}-\mathbf{C z}-\mathbf{p h})_{4}$ as a white powder and after grinding used to prepare a $\mathrm{KBr}$ pellet. The following spectral changes upon grinding are found in the range between 1700 and $1200 \mathrm{~cm}^{-1}$ where the $\mathrm{C}-\mathrm{C}$ stretching vibrations of the conjugated backbone appear: (i) The $\nu$ (CC) mode of the carbazole rings downshifts from 1634 to 1626 $\mathrm{cm}^{-1}$. (ii) An intensity decrease of the $\nu(\mathrm{CC})$ mode of the phenyl rings (observed at $1626 \mathrm{~cm}^{-1}$ ). (iii) A strong decrease of the intense Raman band that appears at $1250 \mathrm{~cm}^{-1}$, which is related to $\mathrm{CC}$ stretching vibrations and $\mathrm{CH}$ bending modes of the carbazoles. (iv) A disappearance of the $\nu(\mathrm{CN})$ band observed at $2248 \mathrm{~cm}^{-1}$ in the white powder, which is very similar to that observed in nonconjugated nitriles (see Figure S15). These experimental changes are in good agreement with the DFT-calculated Raman spectral evolution from $(m-\mathbf{C z}-$ ph) ${ }_{4}$ cyclic oligomer to the open linear oligomers and isolated monomer (see Figure 8b). Our data shows that the cyclophane macrocycle/open linear/monomer transformation involves an aromaticity loss of the carbazole units and the disappearance of the nonconjugated scenario of the $\mathrm{CN}$ groups [note that the
$\mathrm{CN}$ groups in $\sigma$-bonded cyclic oligomers are connected to bridgehead $\mathrm{C}\left(\mathrm{sp}^{3}\right)$ atoms that evolve to $\mathrm{sp}^{2}$ atoms in the monomer]; this is in good agreement with the DFT-calculated bond length evolution going from $(\boldsymbol{m}-\mathbf{C z}-\mathbf{p h})_{4}$ to open linear oligomers to isolated monomer (see Figures S16 and S17).

In summary, this data reveals that (i) $\mathrm{C}-\mathrm{C}$ bond dissociation is favored in solid state when compared to solution, (ii) the dissociation mechanism in meta-substituted systems going from the cyclic oligomers to the isolated monomers implies the formation of open-chain (staircase) oligomers with unpaired electrons at the ends, and (iii) metasubstituted aggregates are harder to dissociate in solid state when compared to para-substituted homologues.

\section{CONCLUSIONS}

This work provides a deep understanding of the structural and electronic parameters controlling the formation of macrocyclic oligomers by self-assembly of biradicaloid carbazole-based systems. Our results demonstrate that oligomerization behavior is strongly modulated by the DCM substitution position and therefore considerably affects their dynamic covalent properties. A stronger biradical character is predicted when DCM is inserted in the meta position of the carbazole unit when compared to the para position, whereas the $\mathrm{N}$ substitution has a negligible effect. As a result, more stable cyclophane macrocycles with attractive $\pi-\pi$ interactions and shorter $\mathrm{C}-\mathrm{C}$ bond bridging are obtained in $\boldsymbol{m}$-Cz-ph when compared to $\boldsymbol{p}$-Cz-alkyl. In fact, although $\boldsymbol{p}$-Cz-alkyl shows a dynamic monomer/cyclic oligomer transformation both in solution and solid state, for $\boldsymbol{m}$-Cz-ph cyclic oligomers, $\mathrm{C}-\mathrm{C}$ bond dissociation by external stimuli is suppressed in solution although favored in solid state through the formation of intermediate staircase oligomers with unpaired electrons at the ends. Therefore, the strong chromic effect from white to purple/deep blue in response to soft external stimuli (i.e., temperature, grinding) can be adequately modulated upon appropriate changes in the substitution pattern position. We believe that this study helps to identify new potential design strategies for stimuli-responsive materials.

\section{EXPERIMENTAL SECTION AND COMPUTATIONAL METHODS}

Electronic absorption spectra were recorded on an Agilent 8453 spectrophotometer (the UV-vis region) and a Varian 
Cary 5000 spectrophotometer (the UV-vis-NIR region). Variable-temperature UV-vis-NIR absorption spectra were recorded in an optical cryostat Optistat DN (Oxford Instruments).

Fourier-transform infrared (FT-IR) spectra of pure solid samples (64 scans, $4 \mathrm{~cm}^{-1}$ ) were recorded with a Golden Gate Single Reflection Diamond ATR System (Graseby Specac) fitted into a Bruker Vertex 70 FT-IR spectrometer. Variabletemperature FT-IR measurements were carried out in a Specac $\mathrm{P} / \mathrm{N} 21000$ cell operating within the temperature range 77$523 \mathrm{~K}$. During the experiments, the temperature was stabilized within $\pm 0.1 \mathrm{~K}$. It was necessary to wait between 10 and $15 \mathrm{~min}$ for the sample to achieve a uniform temperature. Compounds were ground in a pestle and mortar to a powder and pressed in $\mathrm{KBr}$ pellets.

FT-Raman spectra were recorded in an FT-Raman accessory kit (RamII) linked to a Bruker Vertex 70 spectrometer. A continuous-wave $\mathrm{Nd}-\mathrm{YAG}$ laser working at $1064 \mathrm{~nm}$ was employed for excitation. A germanium detector operating at liquid nitrogen temperature was used. Raman radiation was collected in a back-scattering configuration with a standard spectral resolution of $4 \mathrm{~cm}^{-1}$. Scans (3000-4000) were averaged for each spectrum to optimize the signal-to-noise ratio.

Calculations were performed in the gas phase using the Gaussian 09 program. ${ }^{19}$ Simulations were carried out in the framework of the density functional theory (DFT) using the exchange-correlation functional $(\mathrm{M} 06-2 \mathrm{X})^{20}$ and the 6$31 \mathrm{G}(\mathrm{d}, \mathrm{p})^{21}$ basis set. Note that M06-2X is known to include (at least partly) intra- and intermolecular noncovalent interactions, which prompted its use here for isolated and aggregated structures. ${ }^{22}$ To simulate the singlet open-shell structures by DFT, we imposed the broken-symmetry solution with the keyword guess = mix and using an unrestricted wavefunction. For the calculation of the triplet states, standard unrestricted calculations were also done. For the study of relative energies between resonance forms of the ground electronic state in the isolated monomer (i.e., the energy difference between the open-shell and closed-shell singlet states and open-shell singlet and triplet states), the M06 suite of functionals was complementarily used to bracket the effect of the functional choice. These functionals differ in the amount of the exact exchange introduced, with M06 including 27\% of the HF exchange, M06-2X including 54\%, and M06-HF including $100 \%$. $^{23}$

For those aggregates with available X-ray structures [i.e., ( $p$ Cz-alkyl $)_{4}$ and $(\boldsymbol{m} \text {-Cz-ph })_{3}$ cyclic oligomers], their geometry optimizations were also performed using the B3LYP-D3 and M06-2X-D3 functionals, which include an explicit correction for dispersion. ${ }^{24}$ We found that the M06-2X functional, though lacking an explicit dispersion correction, provides quantitative agreement with the experimental data compared to B3LYP-D3 and gives very similar results to those obtained with M06-2XD3 (see Figures S7 and S8 in the Supporting Information). These results confirm that the M06-2X functional seems appropriate for evaluating the properties and structures of the carbazole-based aggregates under study.

To gain deeper insight into the role played by the solvent in the aggregate structural properties, the optimum structures and stability of $(\boldsymbol{p} \text {-Cz-alkyl })_{4}$ and $(\boldsymbol{m} \text {-Cz-ph })_{4}$ cyclic oligomers were determined in toluene, chloroform, $o$-dichlorobenzene, and dimethyl sulfoxide solution using the PCM method ${ }^{25}$ (see Table S3 in the Supporting Information).
Besides, all calculations aiming to disclose the diradical character were performed using the ORCA 4.0 program. ${ }^{26} \mathrm{We}$ have carried out an analysis based on the fractional occupation number weighted density (FOD) whose main feature is to provide a robust and cost-effective information on the localization of "hot" electrons (strongly correlated and chemically active) in a molecule. ${ }^{27}$ We used the FOD analysis for a quantitative description of the open-shell singlet biradical character of these ground-state organic molecules. ${ }^{17}$ The number of hot electrons, $N_{\mathrm{FOD}}$, was calculated at the FT$\mathrm{TPSS}^{28} /$ def2-TZVP ${ }^{29}$ level at a default electronic temperature $\left(T_{\mathrm{el}}\right)$ of $5000 \mathrm{~K}$. The isocontour value of the $\rho^{\mathrm{FOD}}$ plots was fixed to $0.005 e \mathrm{bohr}^{-3}$ allowing the systematic comparison between the different systems tackled here.

\section{ASSOCIATED CONTENT}

\section{Supporting Information}

The Supporting Information is available free of charge on the ACS Publications website at DOI: 10.1021/acsomega.8b03418.

Chemical structures of carbazole-based systems under study; additional DFT-calculated data for carbazolebased monomers; DFT-calculated data for $(\boldsymbol{p} \text {-Cz-alkyl })_{4}$ and $(\boldsymbol{m}-\mathbf{C z}-\mathbf{p h})_{3}$ cyclic oligomers; DFT-calculated data for $(\boldsymbol{p} \text {-Cz-alkyl })_{4}$ and $(\boldsymbol{m} \text {-Cz-ph })_{4}$ in different solvents; DFT-calculated data for open linear oligomers; UV-vis absorption spectra of $(\boldsymbol{m}-\mathbf{C z}-\mathbf{p h})_{4}$ and $(\boldsymbol{m}-\mathbf{C z}-\mathbf{p h})_{3}$; experimental and theoretical IR spectra; additional experimental Raman spectra (PDF)

\section{AUTHOR INFORMATION}

\section{Corresponding Author}

*E-mail: carmenrd@uma.es. ORCID ${ }^{\circ}$

Juan Carlos Sancho-García: 0000-0003-3867-1697

Hongxiang Li: 0000-0002-1032-1583

Daisuke Sakamaki: 0000-0001-6503-1607

Shu Seki: 0000-0001-7851-4405

M. Carmen Ruiz Delgado: 0000-0001-8180-7153

Notes

The authors declare no competing financial interest.

\section{ACKNOWLEDGMENTS}

The work at the University of Málaga was funded by the MINECO (CTQ2015-66897) and Junta de Andalucía (P09FQM-4708) projects. The work at the University of Alicante was supported by the MINECO (CTQ2014-55073) and Generalitat Valenciana (AICO/2018/175) projects. The authors thankfully acknowledge the computer resources, technical expertize, and assistance provided by the SCBI (Supercomputing and Bioinformatics) centre of the University of Malaga.

\section{REFERENCES}

(1) (a) Kamada, K.; Ohta, K.; Kubo, T.; Shimizu, A.; Morita, Y.; Nakasuji, K.; Kishi, R.; Ohta, S.; Furukawa, S.-i.; Takahashi, H.; Nakano, M. Strong Two-Photon Absorption of Singlet Diradical Hydrocarbons. Angew. Chem., Int. Ed. 2007, 46, 3544-3546. (b) Nakano, M.; Kishi, R.; Nitta, T.; Kubo, T.; Nakasuji, K.; Kamada, K.; Ohta, K.; Champagne, B.; Botek, E.; Yamaguchi, K. Second Hyperpolarizability $(\gamma)$ of Singlet Diradical System: Dependence of $\gamma$ on the Diradical Character. J. Phys. Chem. A 2005, 109, 
885-891. (c) Kishida, H.; Hibino, K.; Nakamura, A.; Kato, D.; Abe, J. Third-order nonlinear optical properties of a $\pi$-conjugated biradical molecule investigated by third-harmonic generation spectroscopy. Thin Solid Films 2010, 519, 1028-1030.

(2) (a) Shimizu, A.; Kubo, T.; Uruichi, M.; Yakushi, K.; Nakano, M.; Shiomi, D.; Sato, K.; Takui, T.; Hirao, Y.; Matsumoto, K.; Kurata, H.; Morita, Y.; Nakasuji, K. Alternating Covalent Bonding Interactions in a One-Dimensional Chain of a Phenalenyl-Based Singlet Biradical Molecule Having Kekulé Structures. J. Am. Chem. Soc. 2010, 132, 14421-14428. (b) Kubo, T.; Goto, Y.; Uruichi, M.; Yakushi, K.; Nakano, M.; Fuyuhiro, A.; Morita, Y.; Nakasuji, K. Synthesis and Characterization of Acetylene-Linked Bisphenalenyl and Metallic-Like Behavior in Its Charge-Transfer Complex. Chem. - Asian J. 2007, 2, $1370-1379$.

(3) (a) Kato, D.; Inoue, K.; et al. Synthesis and Magnetic Properties of Conjugated Radical Polymer. Chem. Lett. 2008, 37, 694-695. (b) Hiroto, S.; Furukawa, K.; Shinokubo, H.; Osuka, A. Synthesis and Biradicaloid Character of Doubly Linked Corrole Dimers. J. Am. Chem. Soc. 2006, 128, 12380-12381. (c) Zhu, X.; Tsuji, H.; Nakabayashi, K.; Ohkoshi, S.-i.; Nakamura, E. Air- and Heat-Stable Planar Tri-p-quinodimethane with Distinct Biradical Characteristics. J. Am. Chem. Soc. 2011, 133, 16342-16345.

(4) Muller, P. Glossary of terms used in physical organic chemistry (IUPAC Recommendations 1994). Pure Appl. Chem. 1994, 66, 10771184.

(5) (a) Kubo, T.; Shimizu, A.; Sakamoto, M.; Uruichi, M.; Yakushi, K.; Nakano, M.; Shiomi, D.; Sato, K.; Takui, T.; Morita, Y.; Nakasuji, K. Synthesis, Intermolecular Interaction, and Semiconductive Behavior of a Delocalized Singlet Biradical Hydrocarbon. Angew. Chem., Int. Ed. 2005, 44, 6564-6568. (b) Kolc, J.; Michl, J. pi.,.pi.Biradicaloid hydrocarbons. Pleiadene family. I. Photochemical preparation from cyclobutene precursors. J. Am. Chem. Soc. 1973, 95, 7391-7401. (c) Abe, M. Diradicals. Chem. Rev. 2013, 113, 70117088.

(6) (a) Dewar, M. J. S.; Healy, E. Ab initio study of the chair cope rearrangement of 1,5-hexadiene. Chem. Phys. Lett. 1987, 141, 521524. (b) Li, Z.; Hou, Y.; Li, Y.; Hinz, A.; Chen, X. Biradicaloid and Zwitterion Reactivity of Dicarbondiphosphide Stabilized with NHeterocyclic Carbenes. Chem. - Eur. J. 2018, 24, 4849-4855.

(7) Thomas, H. M.; Kumari, H.; Maddalena, J.; Mayhan, C. M.; Ellis, L. T.; Adams, J. E.; Deakyne, C. A. Conformational preference and dynamics of pyrogallol[4]arene: stability, interconversion, and solvent influence. Supramol. Chem. 2018, 30, 520-532.

(8) Huang, J.; Kertesz, M. Intermolecular Covalent $\pi-\pi$ Bonding Interaction Indicated by Bond Distances, Energy Bands, and Magnetism in Biphenalenyl Biradicaloid Molecular Crystal. J. Am. Chem. Soc. 2007, 129, 1634-1643.

(9) Zafra, J. L.; Qiu, L.; Yanai, N.; Mori, T.; Nakano, M.; Alvarez, M. P.; Navarrete, J. T. L.; Gómez-García, C. J.; Kertesz, M.; Takimiya, K.; Casado, J. Reversible Dimerization and Polymerization of a Janus Diradical To Produce Labile C-C Bonds and Large Chromic Effects. Angew. Chem., Int. Ed. 2016, 55, 14563-14568.

(10) (a) Rowan, S. J.; Cantrill, S. J.; Cousins, G. R. L.; Sanders, J. K. M.; Stoddart, J. F. Dynamic Covalent Chemistry. Angew. Chem., Int. Ed. 2002, 41, 898-952. (b) Okino, K.; Hira, S.; Inoue, Y.; Sakamaki, D.; Seki, S. The Divergent Dimerization Behavior of N-Substituted Dicyanomethyl Radicals: Dynamically Stabilized versus Stable Radicals. Angew. Chem., Int. Ed. 2017, 56, 16597-16601.

(11) Lehn, J.-M. From supramolecular chemistry towards constitutional dynamic chemistry and adaptive chemistry. Chem. Soc. Rev. 2007, 36, 151-160.

(12) (a) Stalindurai, K.; Gokula Krishnan, K.; Nagarajan, E. R.; Ramalingan, C. Experimental and theoretical studies on new 7-(3,6di-tert-butyl-9H-carbazol-9-yl)-10-alkyl-10H-phenothiazine-3-carbaldehydes. J. Mol. Struct. 2017, 1130, 633-643. (b) Ho, K. W.; Ariffin, A. Synthesis, photophysical, and electrochemical properties of wide band gap tetraphenylsilane-carbazole derivatives: Effect of the substitution position and naphthalene side chain. Russ. J. Phys. Chem. A 2016, 90, 2590-2599. (c) Maeda, C.; Yoneda, T.; Aratani,
N.; Yoon, M.-C.; Lim, J. M.; Kim, D.; Yoshioka, N.; Osuka, A. Synthesis of Carbazole-Containing Porphyrinoids by a Multiple Annulation Strategy: A Core-Modified and $\pi$-Expanded Porphyrin. Angew. Chem., Int. Ed. 2011, 50, 5691-5694.

(13) Wang, D.; Capel Ferrón, C.; Li, J.; Gámez-Valenzuela, S.; Ponce Ortiz, R.; López Navarrete, J. T.; Hernández Jolín, V.; Yang, X.; Peña Álvarez, M.; García Baonza, V.; Hartl, F.; Ruiz Delgado, M. C.; Li, H. New Multiresponsive Chromic Soft Materials: Dynamic Interconversion of Short 2,7-Dicyanomethylenecarbazole-Based Biradicaloid and the Corresponding Cyclophane Tetramer. Chem. - Eur. J. 2017, 23, 13776-13783.

(14) Kobashi, T.; Sakamaki, D.; Seki, S. N-Substituted Dicyanomethylphenyl Radicals: Dynamic Covalent Properties and Formation of Stimuli-Responsive Cyclophanes by Self-Assembly. Angew. Chem., Int. Ed. 2016, 55, 8634-8638.

(15) Peterson, J. P.; Geraskina, M. R.; Zhang, R.; Winter, A. H. Effect of Substituents on the Bond Strength of Air-Stable Dicyanomethyl Radical Thermochromes. J. Org. Chem. 2017, 82, 6497-6501.

(16) Luo, D.; Lee, S.; Zheng, B.; Sun, Z.; Zeng, W.; Huang, K.-W.; Furukawa, K.; Kim, D.; Webster, R. D.; Wu, J. Indolo[2,3b]carbazoles with tunable ground states: how Clar's aromatic sextet determines the singlet biradical character. Chem. Sci. 2014, 5, 49444952.

(17) (a) Grimme, S.; Hansen, A. A Practicable Real-Space Measure and Visualization of Static Electron-Correlation Effects. Angew. Chem., Int. Ed. 2015, 54, 12308-12313. (b) Bauer, C. A.; Hansen, A.; Grimme, S. The Fractional Occupation Number Weighted Density as a Versatile Analysis Tool for Molecules with a Complicated Electronic Structure. Chem. - Eur. J. 2017, 23, 6150-6164.

(18) González-Cano, R. C.; Di Motta, S.; Zhu, X.; López Navarrete, J. T.; Tsuji, H.; Nakamura, E.; Negri, F.; Casado, J. Carbon-Bridged Phenylene-Vinylenes: On the Common Diradicaloid Origin of Their Photonic and Chemical Properties. J. Phys. Chem. C 2017, 121, 23141-23148.

(19) Frisch, M. J.; Trucks, G. W.; Schlegel, H. B.; Scuseria, G. E.; Robb, M. A.; Cheeseman, J. R.; Scalmani, G.; Barone, V.; Petersson, G. A.; Nakatsuji, H.; Li, X.; Caricato, M.; Marenich, A. V.; Bloino, J.; Janesko, B. G.; Gomperts, R.; Mennucci, B.; Hratchian, H. P.; Ortiz, J. V.; Izmaylov, A. F.; Sonnenberg, J. L.; Williams; Ding, F.; Lipparini, F.; Egidi, F.; Goings, J.; Peng, B.; Petrone, A.; Henderson, T.; Ranasinghe, D.; Zakrzewski, V. G.; Gao, J.; Rega, N.; Zheng, G.; Liang, W.; Hada, M.; Ehara, M.; Toyota, K.; Fukuda, R.; Hasegawa, J.; Ishida, M.; Nakajima, T.; Honda, Y.; Kitao, O.; Nakai, H.; Vreven, T.; Throssell, K.; Montgomery, J. A., Jr.; Peralta, J. E.; Ogliaro, F.; Bearpark, M. J.; Heyd, J. J.; Brothers, E. N.; Kudin, K. N.; Staroverov, V. N.; Keith, T. A.; Kobayashi, R.; Normand, J.; Raghavachari, K.; Rendell, A. P.; Burant, J. C.; Iyengar, S. S.; Tomasi, J.; Cossi, M.; Millam, J. M.; Klene, M.; Adamo, C.; Cammi, R.; Ochterski, J. W.; Martin, R. L.; Morokuma, K.; Farkas, O.; Foresman, J. B.; Fox, D. J.;et al. Gaussian 09, revision C.01; Gaussian Inc.: Wallingford, CT, 2009.

(20) Zhao, Y.; Truhlar, D. G. Density Functionals with Broad Applicability in Chemistry. Acc. Chem. Res. 2008, 41, 157-167.

(21) (a) Hehre, W. J.; Ditchfield, R.; Pople, J. A. Self-Consistent Molecular Orbital Methods. XII. Further Extensions of GaussianType Basis Sets for Use in Molecular Orbital Studies of Organic Molecules. J. Chem. Phys. 1972, 56, 2257-2261. (b) Francl, M. M.; Pietro, W. J.; Hehre, W. J.; Binkley, J. S.; Gordon, M. S.; DeFrees, D. J.; Pople, J. A. Self-consistent molecular orbital methods. XXIII. A polarization-type basis set for second-row elements. J. Chem. Phys. 1982, 77, 3654-3665.

(22) Zhao, Y.; Truhlar, D. G. The M06 suite of density functionals for main group thermochemistry, thermochemical kinetics, noncovalent interactions, excited states, and transition elements: two new functionals and systematic testing of four M06-class functionals and 12 other functionals. Theor. Chem. Acc. 2008, 120, 215-241.

(23) (a) Zhao, Y.; Truhlar, D. G. A new local density functional for main-group thermochemistry, transition metal bonding, thermochem- 
ical kinetics, and noncovalent interactions. J. Chem. Phys. 2006, 125, No. 194101. (b) Zhao, Y.; Truhlar, D. G. Density Functional for Spectroscopy: No Long-Range Self-Interaction Error, Good Performance for Rydberg and Charge-Transfer States, and Better Performance on Average than B3LYP for Ground States. J. Phys. Chem. A 2006, $110,13126-13130$.

(24) Grimme, S.; Antony, J.; Ehrlich, S.; Krieg, H. J. Chem. Phys. 2010, 132, No. 154104.

(25) Tomasi, J.; Persico, M. Chem. Rev. 1994, 94, 2027-2094.

(26) Neese, F. The ORCA program system. Wiley Interdiscip. Rev.: Comput. Mol. Sci. 2012, 2, 73-78.

(27) Pérez-Guardiola, A.; Sandoval-Salinas, M. E.; Casanova, D.; San-Fabián, E.; Pérez-Jiménez, A. J.; Sancho-García, J. C. The role of topology in organic molecules: origin and comparison of the radical character in linear and cyclic oligoacenes and related oligomers. Phys. Chem. Chem. Phys. 2018, 20, 7112-7124.

(28) Tao, J.; Perdew, J. P.; Staroverov, V. N.; Scuseria, G. E. Climbing the Density Functional Ladder: Nonempirical MetaGeneralized Gradient Approximation Designed for Molecules and Solids. Phys. Rev. Lett. 2003, 91, No. 146401.

(29) Weigend, F.; Ahlrichs, R. Balanced basis sets of split valence, triple zeta valence and quadruple zeta valence quality for $\mathrm{H}$ to $\mathrm{Rn}$ : Design and assessment of accuracy. Phys. Chem. Chem. Phys. 2005, 7, $3297-3305$ 